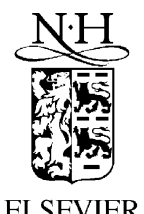

ELSEVIER

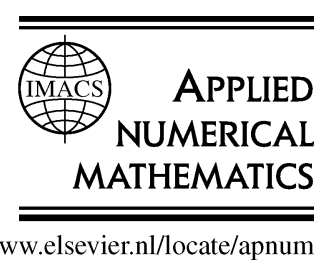

www.elsevier.nl/locate/apnum

\title{
Solution of nonlinearly curvature driven evolution of plane curves
}

\author{
Karol Mikula ${ }^{\mathrm{a}, *}$, Daniel Ševčovič ${ }^{\mathrm{b}, 1}$ \\ a Department of Mathematics, Slovak Technical University, Radlinského 11, 81368 Bratislava, Slovak Republic \\ b Institute of Applied Mathematics, Faculty of Mathematics and Physics, Comenius University, \\ 84215 Bratislava, Slovak Republic
}

\begin{abstract}
The evolution of plane curves obeying the equation $v=\beta(k)$, where $v$ is normal velocity and $k$ curvature of the curve is studied. Morphological image and shape multiscale analysis of Alvarez, Guichard, Lions and Morel and affine invariant scale space of curves introduced by Sapiro and Tannenbaum as well as isotropic motions of plane phase interfaces studied by Angenent and Gurtin are included in the model. We introduce and analyze a numerical scheme for solving the governing equation and present numerical experiments. ๑ 1999 Elsevier Science B.V. and IMACS. All rights reserved.

Keywords: Curve evolution; Image and shape multiscale analysis; Phase interface; Nonlinear degenerate parabolic equations; Numerical solution
\end{abstract}

\section{Introduction}

The goal of this paper is to investigate the evolution of closed smooth plane curves $\Gamma: \mathbb{R} / \mathbb{Z} \rightarrow \mathbb{R}^{2}$. By contrast to the curve shortening flow studied in $[1,7,14,17,18]$, we assume that the normal velocity of the curve $\Gamma$ at its point $x$ is a nonlinear function of the curvature $k$ of $\Gamma$ at $x$. More precisely, we study the evolution of plane curves obeying the geometrical equation

$$
v=\beta(k),
$$

where $v$ is the normal velocity of evolving curves and $\beta: \mathbb{R}_{0}^{+} \rightarrow \mathbb{R}_{0}^{+}$is a smooth function. As a typical example one can consider a function $\beta(k)=k^{m}$, where $m>0$. Throughout this paper we adopt a convention according to which the curvature $k$ of a curve $\Gamma$ is always nonnegative whereas the normal vector $N$ may change its orientation with respect to the tangent vector $T$.

\footnotetext{
*Corresponding author. E-mail: mikula@vox.svf.stuba.sk.

${ }^{1}$ E-mail: sevcovic@fmph.uniba.sk. 
The geometrical equations like (0.1) are capable of describing various phenomena in physics, material sciences, computer vision, robotics and artificial intelligence. There are two main fields, in which the evolution of a plane curve plays an important role: (a) the multiscale analysis of images and shapes closely related to signal smoothing, edge detection and image representation (see, e.g., [3,19,27]); (b) the Stefan problem with surface tension and related interface motion models (see, e.g., [8,24,29]).

In the context of image processing, so-called morphological image multiscale analysis is widely used. This analysis is represented by a viscosity solution $[13,16,12]$ of the following nonlinear degenerate parabolic equation in a two-dimensional rectangular domain

$$
v_{t}=|\nabla v| g(\operatorname{div}(\nabla v /|\nabla v|)),
$$

where $g$ is a nondecreasing function [2,3,21]. It is a generalization of the so-called level set equation [25, 30 ] used for the classical mean curvature flow. The initial condition for $(0.2)$ corresponds to the processed image and the solution $v$ to its scaling version. In many situations, silhouettes (boundaries of distinguished shapes) in the image correspond to level lines of $v$. The morphological image multiscale analysis then leads to the silhouettes motion obeying the equation of the form $(0.1)$. In the vision theory, affine invariant scale space has special conceptual and practical importance [2,28]. It is natural generalization of the linear curve shortening flow, and is given by $(0.1)$ with $\beta(k)=k^{1 / 3}$. The active contours models (snakes) and curvature-based multiscale shape representation, related to edge detection, image segmentation and recognition, are other important fields in which geometrical equations are widely used [20,22].

In the context of multiphase thermomechanics with interfacial structure the plane curve evolution is a natural model for the motion of phase interfaces. The isotropic version of the theory of Angenent and Gurtin [8,9] has the form of equation (0.1). In this case, the nonlinearity expresses the dependence of the kinetic coefficient on the normal velocity (see $[8,(4.11)]$ ). For example, if the dependence is linear then we have $\beta(k)=k^{1 / 2}$. Under additional assumptions, a model corresponding to classical (i.e., anisotropic) curve shortening flow is derived and studied in [8, (4.13)]; for numerical approximation in this case we refer to $[15,24]$. If $\beta$ is a strictly increasing function equation $(0.1)$ has been studied in $[5,6]$ as a model of curve evolution on arbitrary surfaces.

In the present paper, we suggest a new computational method for solving geometrical equation (0.1). The aim is to represent equation $(0.1)$ by a so-called intrinsic heat equation governing the evolution of plane curves with the normal velocity obeying equation (0.1). Such a representation of the curve evolution is found for a general function $\beta$ using an appropriate curve parameterization. In Section 1 we make use of the "Eulerian transformation" of the intrinsic heat equation (1.3) into a degenerate evolution partial differential equation (1.10) with spatial variable being independent on time and varying on a fixed interval. This equation is a generalization of the corresponding equation studied by Dziuk in [14]. The "intrinsic property" of the governing equation (1.10) causes that the spatial parameterization step is not involved in the approximation scheme and therefore only the spatial position of points of a curve $\Gamma$ and the curvature of $\Gamma$ play the role in the discretization scheme suggested in Section 4 . In other words, given a discrete polygonal curve one can compute its evolution without knowing the normalized parameterization of the initial curve. The behavior of homothetic solutions is studied in Section 2 . In Section 3 we prove some a-priori estimates of a smooth solution, which, in particular, imply the curve shortening property of the governing equation (1.10) which ensures, in some way, the stability of the method. The same property is proven for the time discretization scheme (4.1) in Section 4. In Section 5 the proposed numerical scheme is carefully tested by various examples of the nonlinear curvature driven 
evolution (0.1). We present a comparison of the numerical results with the exact homothetic solutions. In this section we also perform a comparison with previous results obtained by a conceptually different method introduced in [23]. It is worthwhile noting that the method of [23] can be applied only for the evolution of convex curves whereas the new method suggested in this paper is capable of capturing the nonlinear evolution of both convex as well as nonconvex curves.

Notice that coefficients in Eq. (1.10) may develop singularities either due to vanishing of $x_{u}$, or, by contrast to the case $\beta(k)=k$ studied in [14], also due to the presence of the extremal values of the curvature $k=0$ or $k=\infty$. Moreover, Eq. (1.10) is written in a non-divergence form. This feature make the analysis particularly difficult. Therefore the careful analysis of the convergence as well as error estimates of the suggested approximation scheme are still open problems.

\section{Governing equations}

\subsection{Parameterization of a plane curve}

Let $\Gamma$ be a smooth curve in the plane $\mathbb{R}^{2}$. By this we mean that $\Gamma$ can be parameterized by a $C^{2}$ smooth function $x: \mathbb{R} / \mathbb{Z} \rightarrow \mathbb{R}^{2}$ such that

$$
\Gamma=\{x(u), u \in[0,1]\} .
$$

We will henceforth write $\Gamma=\operatorname{Image}(x)$. To describe the time evolution $\left\{\Gamma^{t}\right\}, t \in\left[0, T_{\max }\right)$, of a curve $\Gamma^{0}$ we adopt the notation

$$
\Gamma^{t}=\{x(u, t), u \in[0,1]\}, \quad t \in\left[0, T_{\max }\right),
$$

where $x \in C^{2}\left(\mathbb{R} / \mathbb{Z} \times\left[0, T_{\max }\right), \mathbb{R}^{2}\right)$. Obviously, any plane curve $\Gamma$ admits various other parameterizations. Henceforth, the parameter $s$ will always refer to the arc-length parameter of a plane curve $\Gamma$.

Example 1.1. Consider another parameterization $s_{*}$ of a curve $\Gamma=\operatorname{Image}(x)$. Then it is easy to verify that $\operatorname{det}\left[\partial x / \partial s_{*}, \partial^{2} x / \partial s_{*}^{2}\right]=\phi^{\prime}(s)^{-3} \operatorname{det}\left[\partial x / \partial s, \partial^{2} x / \partial s^{2}\right]$, where $s_{*}=\phi(s)$. As $k=\mid \operatorname{det}[\partial x / \partial s$, $\left.\partial^{2} x / \partial s^{2}\right] \mid$ we have

$$
\left|\operatorname{det}\left[\partial x / \partial s_{*}, \partial^{2} x / \partial s_{*}^{2}\right]\right|=1
$$

provided that the new parameterization $s_{*}=\phi(s)$ has the property $\mathrm{d} s_{*}=\vartheta(s) \mathrm{d} s$ where $\vartheta=k^{1 / 3}$. A parameterization of a plane curve satisfying Eq. (1.2) is referred to as the affine arc-length (see [28]).

Throughout the paper we will use both notations $x_{\xi}$ as well as $\partial x / \partial \xi$ in order to denote the partial derivative of $x$ with respect to a variable $\xi$.

\subsection{Intrinsic heat equation}

The aim of this paper is to investigate the evolution of plane curves $\left\{\Gamma^{t}\right\}$ undergoing the intrinsic heat equation

$$
\frac{\partial x}{\partial t}=\frac{\partial^{2} x}{\partial s_{*}^{2}},
$$


where $s_{*}$ is a new parameterization of a curve $\Gamma^{t}$ obeying the law

$$
\mathrm{d} s_{*}=\vartheta(s) \mathrm{d} s .
$$

We will seek for a function $\vartheta$ such that the normal component of the curve-flow velocity $v$ satisfies the equation $v=\beta(k)$. To this end, let us transform Eq. (1.3) using the arc-length parameterization. We obtain

$$
\frac{\partial x}{\partial t}=\frac{1}{\vartheta(s)} \frac{\partial}{\partial s}\left(\frac{1}{\vartheta(s)} \frac{\partial x}{\partial s}\right)=\frac{1}{\vartheta^{2}(s)} k N-\frac{\vartheta^{\prime}(s)}{\vartheta^{3}(s)} T,
$$

where $T$ is the unit tangent vector, $T=x_{s}$ and $N$ is the unit normal vector satisfying Frenet's formula $T_{s}=k N$. Hence the normal velocity $v=\left(x_{t}, N\right)$ fulfills Eq. $(0.1)$ iff

$$
\vartheta=k^{1 / 2} \beta(k)^{-1 / 2} \text {. }
$$

If the function $\beta$ has the form $\beta(k)=k^{1 / \alpha}, \alpha>0$, we obtain $\vartheta=k^{(\alpha-1) /(2 \alpha)}$. If $\alpha=1$ then $x_{t}=k N$. On the other hand, if $\alpha=3$ we have $\vartheta=k^{1 / 3}$ and $x_{t}=k^{1 / 3} N-\frac{1}{3}\left(k_{s} / k^{5 / 3}\right) T$. Taking into account Example 1.1 we may conclude that for the affine arc-length parameterization satisfying (1.2) the normal velocity $v$ of a curve $\Gamma$ with the curvature $k$ at a point $x$ satisfies $v=k^{1 / 3}$ (see also [28]).

\subsection{Eulerian form of the governing equation}

It is worthwhile noting that the parameterization $s_{*}$ occurring in (1.3) may depend on time $t$ and its initial position $u$ at $t=0$. This is because of the requirement that the normal velocity should depend on the curvature only as it was prescribed by Eq. (0.1). Thus the evolution of the new parameterization $s_{*}=s_{*}(u, t)$ as well as the arc-length parameterization $s=s(u, t)$ depend on the solution $x$ itself. This feature is similar, in spirit, to the transformation between Lagrangian (material) and Eulerian (spatial) coordinates in the classical mechanics. This is why the intrinsic heat equation (1.3) is not convenient when treating evolution of plane curves numerically. To overcome this difficulty, we rewrite (1.3) into a form involving a parameterization $u$ independent of the time variable $t$ and varying on the fixed interval $[0,1]$.

Let $u \in[0,1]$ be a time independent parameterization of a curve $\Gamma$. Then the arc-length parameterization $s$ of $\Gamma$ is related to $u$ by $\mathrm{d} s=\left|x_{u}\right| \mathrm{d} u$. Furthermore, as $k=\left|x_{s s}\right|$ and

$$
\frac{\partial^{2} x}{\partial s^{2}}=\frac{1}{\left|x_{u}\right|} \frac{\partial}{\partial u}\left(\frac{1}{\left|x_{u}\right|} \frac{\partial x}{\partial u}\right)=\frac{1}{\left|x_{u}\right|^{2}}\left(x_{u u}-\frac{1}{\left|x_{u}\right|^{2}}\left(x_{u}, x_{u u}\right) x_{u}\right)
$$

we have $k=k\left(x_{u}, x_{u u}\right)$, where

$$
k(p, q)=|p|^{-3}\left(|p|^{2}|q|^{2}-(p, q)^{2}\right)^{1 / 2}, \quad p, q \in \mathbb{R}^{2},
$$

where $(\cdot, \cdot)$ denotes the Euclidean scalar product in $\mathbb{R}^{2}$ and the corresponding norm is denoted by $|\cdot|$. Here and after we will assume that a function

(B) $\beta:[0, \infty) \rightarrow[0, \infty)$ is $C^{1}$-smooth on $(0, \infty)$ and is continuous on $[0, \infty)$, $\beta(k)>0$ for $k>0$.

Let us consider a new parameterization $s_{*}$ satisfying $\mathrm{d} s_{*}=\vartheta(s) \mathrm{d} s$, where $\vartheta(s)$ is defined as in (1.5), i.e., $\vartheta=k^{1 / 2} \beta(k)^{-1 / 2}$. To facilitate the notation, let us define scalar valued functions $\theta_{\beta}, G_{\beta}: \mathbb{R}^{2} \times \mathbb{R}^{2} \rightarrow$ $\mathbb{R}_{0}^{+}$,

$$
\theta_{\beta}(p, q)=k(p, q)^{1 / 2} \beta(k(p, q))^{-1 / 2}, \quad G_{\beta}(p, q)=|p| \theta_{\beta}(p, q) .
$$


It is easy to verify that $k(p, q)=|p|^{-3}|\operatorname{det}[p, q]|$ and this is why the functions $G_{\beta}$ and $k$ have the following scaling and affine properties:

$$
G_{\beta}(p, a p+q)=G_{\beta}(p, q), \quad k(p, a p+q)=k(p, q), \quad k(a p, b q)=a^{-2} b k(p, q)
$$

for any $p, q \in \mathbb{R}^{2}, a, b \in \mathbb{R}$.

If $\beta(k)=k^{1 / \alpha}, \alpha>0$, then

$$
G_{\beta}(p, q)=|p|^{(3-\alpha) /(2 \alpha)}\left(|p|^{2}|q|^{2}-(p, q)^{2}\right)^{(\alpha-1) /(4 \alpha)},
$$

and, in addition to (1.8), one has

$$
G_{\beta}(a p, b q)=|a|^{1 / \alpha}|b|^{(\alpha-1) /(2 \alpha)} G_{\beta}(p, q) \text { for any } p, q \in \mathbb{R}^{2}, a, b \in \mathbb{R} .
$$

Now we are in a position to rewrite the intrinsic heat equation (1.3) into so-called "Eulerian form" with a parameterization $u$ varying on fixed interval $[0,1]$ as follows:

$$
\frac{\partial x}{\partial t}=\frac{1}{G_{\beta}\left(x_{u}, x_{u u}\right)} \frac{\partial}{\partial u}\left(\frac{1}{G_{\beta}\left(x_{u}, x_{u u}\right)} \frac{\partial x}{\partial u}\right), \quad(u, t) \in[0,1] \times\left[0, T_{\max }\right) .
$$

The fully nonlinear system of PDEs (1.10) is subject to the initial condition $x(u, 0)=x^{0}(u), u \in[0,1]$, and periodic boundary conditions at $u=0,1$, i.e., $x \in C^{2,1}\left(\mathbb{R} / \mathbb{Z} \times\left[0, T_{\max }\right), \mathbb{R}^{2}\right)$.

\section{Special solutions}

Throughout this section we will restrict ourselves to the case when $\beta(k)=k^{1 / \alpha}, \alpha>0$. We will seek for a solution $x(u, t)$ of (1.10) having the form

$$
x(u, t)=\phi(t) \tilde{x}(u) .
$$

Suppose that $\tilde{x} \in C^{2}\left(\mathbb{R} / \mathbb{Z} ; \mathbb{R}^{2}\right), \tilde{x} \neq 0$, is a solution of the nonlinear eigenvalue problem

$$
-\frac{1}{G_{\beta}\left(\tilde{x}_{u}, \tilde{x}_{u u}\right)} \frac{\partial}{\partial u}\left(\frac{1}{G_{\beta}\left(\tilde{x}_{u}, \tilde{x}_{u u}\right)} \frac{\partial \tilde{x}}{\partial u}\right)=\lambda \tilde{x}, \quad u \in[0,1] .
$$

Clearly, if $\tilde{x} \neq 0$ is a solution of (2.2) then by taking the scalar product in $\left(L^{2}(0,1)\right)^{2}$ of (2.2) with $G_{\beta} \tilde{x}$ we obtain

$$
\lambda=\frac{\int_{0}^{1}\left(1 / G_{\beta}\right)\left|\tilde{x}_{u}\right|^{2}}{\int_{0}^{1} G_{\beta}|\tilde{x}|^{2}}>0 .
$$

Let $\phi$ be a solution of the initial value problem

$$
\frac{\mathrm{d} \phi}{\mathrm{d} t}=-\lambda \phi^{-1 / \alpha}, \quad \phi(0)=\phi_{0}>0 .
$$

Then it should be obvious from the scaling property (1.9) that the function $x(u, t)=\phi(t) \tilde{x}(u)$ is a solution of (1.10) satisfying the initial condition $x(u, 0)=\phi_{0} \tilde{x}(u), u \in[0,1]$. The explicit form of a solution of (2.3) is given by

$$
\phi(t)=\left[\phi_{0}^{(1+\alpha) / \alpha}-\frac{1+\alpha}{\alpha} \lambda t\right]^{\alpha /(\alpha+1)} .
$$


The life-span of a homothetic solution of the form (2.1) is the interval $\left[0, T_{\max }\right)$, where

$$
T_{\max }=\frac{\alpha \phi_{0}^{(1+\alpha) / \alpha}}{(1+\alpha) \lambda} \text {. }
$$

Example 2.1. An ellipse $\widetilde{\Gamma}=\left\{(a \cos (2 \pi u), b \sin (2 \pi u))^{\mathrm{T}}, u \in[0,1]\right\}$ is a solution of (2.2). Then

$$
G_{\beta}\left(\tilde{x}_{u}, \tilde{x}_{u u}\right)=2 \pi(a b)^{(\alpha-1) /(2 \alpha)}\left[a^{2} \sin ^{2}(2 \pi u)+b^{2} \cos ^{2}(2 \pi u)\right]^{(3-\alpha) /(4 \alpha)} .
$$

In the case $a=b$ (i.e., $\widetilde{\Gamma}$ is a circle) and $\alpha>0$ we have $G_{\beta}=2 \pi a^{(\alpha+1) /(2 \alpha)}$ and so $\tilde{x}$ is a solution of (2.2) iff $\lambda=a^{-(\alpha+1) / \alpha}$. If we choose the initial condition $\phi_{0}=1$ (i.e., $\Gamma^{0}=\widetilde{\Gamma}$ ) then the life-span of a solution is $T_{\max }=(\alpha /(\alpha+1)) a^{(\alpha+1) / \alpha}$. On the other hand, if $a \neq b$ and $\alpha=3$ we obtain $G_{\beta}=2 \pi(a b)^{1 / 3}$ and so $\lambda=(a b)^{-2 / 3}$. Then $T_{\max }=\frac{3}{4}(a b)^{2 / 3}$.

By using the phase-space analysis argument, one can show that the only solution of Eq. (2.1) with normalized $\lambda=1$ is either a circle for $0<\alpha \neq 3$, or an ellipse for $\alpha=3$. Thus a function $x \in C^{2,1}\left(\mathbb{R} / \mathbb{Z} \times[0, T), \mathbb{R}^{2}\right)$ of the form $x(u, t)=\phi(t) \tilde{x}(u)$ is a solution of (1.10) iff the family of its images $\Gamma^{t}=\operatorname{Image}(x(\cdot, t)), t \in[0, T)$, are either homothetically shrinking circles for $0<\alpha \neq 3$ or homothetically shrinking ellipses for the case $\alpha=3$. This is consistent with the result obtained by Sapiro and Tannenbaum [28] for the case $\beta(k)=k^{1 / 3}$.

\section{A-priori estimates of solutions}

The goal of this section is to derive a-priori estimates of solutions of the intrinsic heat equation (1.10). We will provide these estimates for the original equation (1.10) as well as for the modified equation

$$
\frac{\partial x}{\partial t}=\frac{1}{G_{\beta, \varepsilon}\left(x_{u}, x_{u u}\right)} \frac{\partial}{\partial u}\left(\frac{1}{G_{\beta, \varepsilon}\left(x_{u}, x_{u u}\right)} \frac{\partial x}{\partial u}\right),
$$

where $G_{\beta, \varepsilon}, \varepsilon \geqslant 0$, is a modification of $G_{\beta}$ such that

$$
G_{\beta, \varepsilon}: \mathbb{R}^{2} \times \mathbb{R}^{2} \rightarrow \mathbb{R} \text { is } C^{1} \text {-smooth, } 0<G_{\beta, \varepsilon}(p, q)<\infty,
$$

(E) $G_{\beta, \varepsilon}(p, a p+q)=G_{\beta, \varepsilon}(p, q)$, for any $p, q \in \mathbb{R}^{2}, a, b \in \mathbb{R}$ and $\varepsilon>0$, and $G_{\beta, \varepsilon}(p, q) \rightarrow G_{\beta, 0}(p, q)=G_{\beta}(p, q)$ as $\varepsilon \rightarrow 0^{+}$for any $p, q \in \mathbb{R}^{2}$.

Definition 3.1. By a nondegenerate evolving curve we mean a function $x \in C^{2,1}\left(\mathbb{R} / \mathbb{Z} \times[0, T), \mathbb{R}^{2}\right)$ such that $0<G_{\beta, \varepsilon}\left(x_{u}(u, t), x_{u u}(u, t)\right)<\infty$ for any $u \in \mathbb{R}, t \in[0, T)$. By a nondegenerate classical solution of Eq. (3.1) we mean a nondegenerate evolving curve $x \in C^{2,1}\left(\mathbb{R} / \mathbb{Z} \times[0, T), \mathbb{R}^{2}\right)$ satisfying (3.1).

Proposition 3.1. Let $x$ be a nondegenerate classical solution of Eq. (3.1), $\varepsilon \geqslant 0$. Then, for each $t \in\left(0, T_{\max }\right)$,

$$
\frac{\mathrm{d}}{\mathrm{d} t} \int_{0}^{1}\left|x_{u}(\cdot, t)\right|+\int_{0}^{1} \omega_{\varepsilon}^{2} k \beta(k)\left|x_{u}(\cdot, t)\right|=0,
$$

where $k=k\left(x_{u}, x_{u u}\right)$ and $\omega_{\varepsilon}=G_{\beta}\left(x_{u}, x_{u u}\right) / G_{\beta, \varepsilon}\left(x_{u}, x_{u u}\right)$ for $\varepsilon>0, \omega_{0}=1$. 
Since $\mathrm{d} s=\left|x_{u}\right| \mathrm{d} u$ we have

$$
\int_{\Gamma^{t}} \omega_{\varepsilon}^{2} k \beta(k) \mathrm{d} s=\int_{0}^{1} \omega_{\varepsilon}^{2} k \beta(k)\left|x_{u}\right| \mathrm{d} u .
$$

Here $k$ stands for the curvature of $\Gamma^{t}$ at a point $x \in \Gamma^{t}$. Therefore, Eq. (3.2) can be rewritten as

$$
\frac{\mathrm{d}}{\mathrm{d} t} \text { Length }\left(\Gamma^{t}\right)+\int_{\Gamma^{t}} \omega_{\varepsilon}^{2} k \beta(k) \mathrm{d} s=0 .
$$

Corollary 3.2. Let $\Gamma^{t}, t \in\left[0, T_{\max }\right)$, where $\Gamma^{t}=\operatorname{Image}(x(\cdot, t))$ be a flow of plane curves where $x$ is a nondegenerate classical solution of (3.1). Then $(\mathrm{d} / \mathrm{d} t)\left|\Gamma^{t}\right| \leqslant 0$. In other words, the length $\left|\Gamma^{t}\right|$ of the curve $\Gamma^{t}$ decreases along the time, i.e., $\left\{\Gamma^{t}\right\}, t \in\left[0, T_{\max }\right)$, is a curve shortening flow.

Proof of Proposition 3.1. Denote $k=k\left(x_{u}, x_{u u}\right), G=G_{\beta, \varepsilon}\left(x_{u}, x_{u u}\right)$ and $\theta=\left|x_{u}\right|^{-1} G$. Then

$$
\begin{aligned}
\left|x_{u}\right|_{t} & =\frac{1}{\left|x_{u}\right|}\left(x_{u t}, x_{u}\right)=\theta\left(x_{u t}, \frac{x_{u}}{G}\right)=\theta\left\{\frac{\mathrm{d}}{\mathrm{d} u}\left(x_{t}, \frac{x_{u}}{G}\right)-\left(x_{t}, \frac{\mathrm{d}}{\mathrm{d} u} \frac{x_{u}}{G}\right)\right\} \\
& =\theta\left\{\frac{\mathrm{d}}{\mathrm{d} u}\left(\frac{1}{G} \frac{\mathrm{d}}{\mathrm{d} u} \frac{x_{u}}{G}, \frac{x_{u}}{G}\right)-\left(x_{t}, G x_{t}\right)\right\}=-\theta^{2}\left|x_{u}\right|\left|x_{t}\right|^{2}+\theta \frac{\mathrm{d}}{\mathrm{d} u}\left\{\left(\frac{1}{\theta^{2}\left|x_{u}\right|} \frac{\mathrm{d}}{\mathrm{d} u} \frac{x_{u}}{\theta\left|x_{u}\right|}, \frac{x_{u}}{\left|x_{u}\right|}\right)\right\} \\
& =-\theta^{2}\left|x_{u}\right|\left|x_{t}\right|^{2}+\theta \frac{\mathrm{d}}{\mathrm{d} u}\left\{\frac{-\theta_{u}}{\theta^{4}\left|x_{u}\right|}\left(\frac{x_{u}}{\left|x_{u}\right|}, \frac{x_{u}}{\left|x_{u}\right|}\right)+\frac{1}{\theta^{3}\left|x_{u}\right|}\left(\frac{\mathrm{d}}{\mathrm{d} u} \frac{x_{u}}{\left|x_{u}\right|}, \frac{x_{u}}{\left|x_{u}\right|}\right)\right\} \\
& =-\theta^{2}\left|x_{u}\right|\left|x_{t}\right|^{2}-\theta \frac{\mathrm{d}}{\mathrm{d} u}\left(\frac{\theta_{u}}{\theta^{4}\left|x_{u}\right|}\right)
\end{aligned}
$$

because

$$
\left(\frac{\mathrm{d}}{\mathrm{d} u} \frac{x_{u}}{\left|x_{u}\right|}, \frac{x_{u}}{\left|x_{u}\right|}\right)=0 .
$$

With regard to (1.6) we have $\left|x_{u}\right|^{2}\left|x_{u u}\right|^{2}-\left(x_{u}, x_{u u}\right)^{2}=k^{2}\left|x_{u}\right|^{6}$. Therefore,

$$
\begin{aligned}
\left|x_{t}\right|^{2} & =\frac{1}{\theta^{2}\left|x_{u}\right|^{2}}\left|\frac{\mathrm{d}}{\mathrm{d} u} \frac{x_{u}}{\theta\left|x_{u}\right|}\right|^{2}=\frac{1}{\theta^{6}\left|x_{u}\right|^{6}}|\theta| x_{u}\left|x_{u u}-\theta_{u}\right| x_{u}\left|x_{u}-\theta \frac{\left(x_{u}, x_{u u}\right)}{\left|x_{u}\right|} x_{u}\right|^{2} \\
& =\frac{1}{\theta^{6}\left|x_{u}\right|^{6}}\left\{\theta^{2}\left[\left|x_{u u}\right|^{2}\left|x_{u}\right|^{2}-\left(x_{u}, x_{u u}\right)^{2}\right]+\theta_{u}^{2}\left|x_{u}\right|^{4}\right\}=k^{2} \theta^{-4}+\frac{\theta_{u}^{2}}{\theta^{6}\left|x_{u}\right|^{2}} .
\end{aligned}
$$

Taking into account (1.7), (3.3) and (3.4) we obtain

$$
\left|x_{u}\right|_{t}=-\left|x_{u}\right| k^{2} \theta^{-2}-\frac{\mathrm{d}}{\mathrm{d} u}\left(\frac{\theta_{u}}{\theta^{3}\left|x_{u}\right|}\right) \text {. }
$$

Finally, as

$$
k^{2} \theta^{-2}=k^{2}\left|x_{u}\right|^{2} G_{\beta, \varepsilon}^{-2}=\frac{k\left|x_{u}\right|^{2}}{\beta(k)} G_{\beta, \varepsilon}^{-2} k \beta(k)=G_{\beta}^{2} G_{\beta, \varepsilon}^{-2} k \beta(k)=\omega_{\varepsilon}^{2} k \beta(k)
$$

we conclude that

$$
\left|x_{u}\right|_{t}=-\omega_{\varepsilon}^{2}\left|x_{u}\right| k \beta(k)-\frac{\mathrm{d}}{\mathrm{d} u}\left(\frac{\theta_{u}}{\theta^{3}\left|x_{u}\right|}\right) .
$$


Since both $x(u, t)$ as well as $\theta(u, t)$ are 1-periodic functions in $u$ Eq. (3.2) follows from (3.5) by integrating over the interval $[0,1]$.

\section{Numerical scheme}

In this section we present a time semi-discretization scheme for solving Eq. (3.1). Let $[0, T]$ be an interval and let $\tau=T / n, n \in \mathbb{N}$, denote the time discretization step. By $x^{i}, i=0,1, \ldots, n$, we denote the approximation of a true solution of (3.1) at time $t=i \tau$, i.e., $x^{i}(\cdot)=x(\cdot, i \tau)$. Let $\varepsilon>0$ be fixed. The idea of the construction of a time discretization scheme is based on approximation of the intrinsic heat equation (1.3) by the backward Euler method

$$
\frac{x^{i}-x^{i-1}}{\tau}=\frac{\partial^{2} x^{i}}{d s_{*}^{2}}, \quad i=1,2, \ldots, n,
$$

where the parameterization $s_{*}$ is computed from the previous time step $x^{i-1}$. The "Eulerian form" of the above scheme reads as follows:

$$
x^{i}-\frac{\tau}{g^{i-1}} \frac{\partial}{\partial u}\left(\frac{1}{g^{i-1}} \frac{\partial x^{i}}{\partial u}\right)=x^{i-1}, \quad i=1,2, \ldots, n,
$$

where $g^{i-1}=G_{\beta, \varepsilon}\left(x_{u}^{i-1}, x_{u u}^{i-1}\right)$ and $x^{0}$ is the initial condition.

In what follows we will investigate the discretization scheme (4.1). We will prove the existence of a sequence $x^{i}, i=1,2, \ldots, n$, as well as we will show that such a discretization of the governing equation (3.1) generates the curve shortening discrete semiflow.

Lemma 4.1. Suppose that $g \in C^{1}(\mathbb{R} / \mathbb{Z} ; \mathbb{R}), g>0, \tau>0$, and $\bar{x} \in C\left(\mathbb{R} / \mathbb{Z} ; \mathbb{R}^{2}\right)$. Then there exists a unique solution $x \in C^{2}\left(\mathbb{R} / \mathbb{Z} ; \mathbb{R}^{2}\right)$ of the equation

$$
x-\frac{\tau}{g} \frac{\partial}{\partial u}\left(\frac{1}{g} \frac{\partial x}{\partial u}\right)=\bar{x} .
$$

Moreover,

$$
\int_{0}^{1} g|x|^{2}+2 \tau \int_{0}^{1} \frac{1}{g}\left|x_{u}\right|^{2} \leqslant \int_{0}^{1} g|\bar{x}|^{2}
$$

and, in particular, $x=0$ whenever $\bar{x}=0$. Finally, if $\bar{x} \in C^{1}\left(\mathbb{R} / \mathbb{Z} ; \mathbb{R}^{2}\right)$ then

$$
\int_{0}^{1}\left|x_{u}\right|+\tau \int_{0}^{1}\left(\frac{k\left|x_{u}\right|}{g}\right)^{2}\left|x_{u}\right| \leqslant \int_{0}^{1}\left|\bar{x}_{u}\right|,
$$

where $k=k\left(x_{u}, x_{u u}\right)$. 
Proof. We first prove the uniqueness of a solution of (4.2). Since (4.2) is a linear nonhomogeneous equation for $x$ the proof follows from (4.3) with $\bar{x}=0$. To prove (4.3) one can take the $L^{2}$ inner product of (4.2) with $g x$ to obtain the estimate

$$
2 \int_{0}^{1} g|x|^{2}+2 \tau \int_{0}^{1} \frac{1}{g}\left|x_{u}\right|^{2}=2 \int_{0}^{1} g(x, \bar{x}) \leqslant \int_{0}^{1} g|x|^{2}+\int_{0}^{1} g|\bar{x}|^{2}
$$

from which inequality (4.3) easily follows.

To prove the existence of a solution of (4.2) one can argue by Fredholm's alternative. Indeed, let $A: C\left(\mathbb{R} / \mathbb{Z} ; \mathbb{R}^{2}\right) \rightarrow C^{2}\left(\mathbb{R} / \mathbb{Z} ; \mathbb{R}^{2}\right)$ be a solution operator for the equation $x_{\text {ии }}+\pi^{2} x=f$, i.e., $x=A f$. Then

$$
A f(u)=-\frac{1}{2 \pi} \int_{0}^{1} \sin (\pi u-\pi s) f(s) \mathrm{d} s+\frac{1}{\pi} \int_{0}^{u} \sin (\pi u-\pi s) f(s) \mathrm{d} s
$$

and this is why the linear operator $A$ is bounded when operating from $C \rightarrow C^{2}$ and is compact as an operator from $C \rightarrow C^{1}$. Let $L$ be a linear operator on $C^{1}\left(\mathbb{R} / \mathbb{Z} ; \mathbb{R}^{2}\right)$ defined as $L x=A\left(g_{u} g^{-1} x_{u}+\right.$ $\left.g^{2} \tau^{-1} x+\pi^{2} x\right)$. Then $L: C^{1} \rightarrow C^{1}$ is compact and therefore $I-L$ is a Fredholm mapping of zero index. As a consequence of (4.3) we have that the kernel of $I-L$ is trivial. Therefore, the equation $x-L x=-A\left(g^{2} \tau^{-1} \bar{x}\right)$ has a solution $x \in C^{1}$. In fact, $x \in C^{2}$ and $x$ solves (4.2).

Finally we prove (4.4). The proof is similar, in technique, to that of Proposition 3.1. Let us denote $\theta=\left|x_{u}\right|^{-1} g, \delta_{\tau} x=(x-\bar{x}) / \tau$. Then, following the lines of the proof of Proposition 3.1 one obtains

$$
\begin{aligned}
\left(\delta_{\tau} x_{u}, \frac{x_{u}}{\left|x_{u}\right|}\right) & =\theta\left(\delta_{\tau} x_{u}, \frac{x_{u}}{g}\right)=\theta\left\{\frac{\mathrm{d}}{\mathrm{d} u}\left(\delta_{\tau} x, \frac{x_{u}}{g}\right)-\left(\delta_{\tau} x, \frac{\mathrm{d}}{\mathrm{d} u} \frac{x_{u}}{g}\right)\right\} \\
& =-\theta^{2}\left|x_{u}\right|\left|\delta_{\tau} x\right|^{2}-\theta \frac{\mathrm{d}}{\mathrm{d} u}\left(\frac{\theta_{u}}{\theta^{4}\left|x_{u}\right|}\right) .
\end{aligned}
$$

Using the same argument as in (3.4) yields

$$
\left|\delta_{\tau} x\right|^{2}=\frac{1}{\theta^{2}\left|x_{u}\right|^{2}}\left|\frac{\mathrm{d}}{\mathrm{d} u} \frac{x_{u}}{\theta\left|x_{u}\right|}\right|^{2}=k^{2} \theta^{-4}+\frac{\theta_{u}^{2}}{\theta^{6}\left|x_{u}\right|^{2}} .
$$

Hence,

$$
\left(\delta_{\tau} x_{u}, \frac{x_{u}}{\left|x_{u}\right|}\right)=-k^{2} \theta^{-2}\left|x_{u}\right|-\frac{\mathrm{d}}{\mathrm{d} u}\left(\frac{\theta_{u}}{\theta^{3}\left|x_{u}\right|}\right) .
$$

On the other hand,

$$
\left(\delta_{\tau} x_{u}, \frac{x_{u}}{\left|x_{u}\right|}\right)=\frac{1}{\tau}\left(x_{u}-\bar{x}_{u}, \frac{x_{u}}{\left|x_{u}\right|}\right)=\frac{1}{\tau}\left(\left|x_{u}\right|-\left(\bar{x}_{u}, \frac{x_{u}}{\left|x_{u}\right|}\right)\right) .
$$

Therefore,

$$
\int_{0}^{1}\left|x_{u}\right|+\tau \int_{0}^{1}\left(\frac{k}{\theta}\right)^{2}\left|x_{u}\right|=\int_{0}^{1}\left(\bar{x}_{u}, \frac{x_{u}}{\left|x_{u}\right|}\right) \leqslant \int_{0}^{1}\left|\bar{x}_{u}\right|
$$

and the proof of the lemma follows. 
We claim that we have assured the existence of a sequence $x^{i} \in C^{2}\left(\mathbb{R} / \mathbb{Z} ; \mathbb{R}^{2}\right), i=0,1, \ldots, n$, generated by the iteration scheme (4.1) provided that $x^{0} \in C^{3}\left(\mathbb{R} / \mathbb{Z} ; \mathbb{R}^{2}\right)$. Indeed, for $\varepsilon>0$ we have $g^{0}>0$ and $g^{0} \in C^{1}$. Now, if $g^{i-1} \in C^{1}$ then according to Lemma 4.1 there exists the unique solution $x^{i} \in C^{2}$ of (4.1). Then

$$
x_{u u}^{i}=\frac{g_{u}^{i-1}}{g^{i-1}} x_{u}^{i}+\frac{\left(g^{i-1}\right)^{2}}{\tau}\left(x^{i}-x^{i-1}\right) .
$$

By the hypothesis (E) and the property (1.8) we may conclude that the function $g^{i}=G_{\beta, \varepsilon}\left(x_{u}^{i}, x_{u u}^{i}\right)=$ $G_{\beta, \varepsilon}\left(x_{u}^{i},\left(g^{i-1}\right)^{2} \tau^{-1}\left(x^{i}-x^{i-1}\right)\right)$ is, in effect, $C^{1}$-smooth and $g^{i}>0$. Then an induction argument enables to conclude that the sequence $x^{i}, i=0,1, \ldots, n$, is well defined and all $x^{i}, i=0,1, \ldots, n$, are $C^{2}$ smooth.

Summarizing the above considerations we obtain the following result.

Proposition 4.2. Let $x^{0} \in C^{3}\left(\mathbb{R} / \mathbb{Z} ; \mathbb{R}^{2}\right)$ and $\varepsilon>0$. Then there exists a unique sequence $x^{i}, i=$ $0,1, \ldots, n$, generated according to the iteration scheme (4.1). Moreover,

$$
\int_{0}^{1}\left|x_{u}^{i}\right|+\tau \int_{0}^{1}\left(\frac{k^{i}\left|x_{u}^{i}\right|}{g^{i-1}}\right)^{2}\left|x_{u}^{i}\right| \leqslant \int_{0}^{1}\left|x_{u}^{i-1}\right| \text { for } i=1,2, \ldots, n,
$$

where $k^{i}=k\left(x_{u}^{i}, x_{u u}^{i}\right)$ and $g^{i-1}=G_{\beta, \varepsilon}\left(x_{u}^{i-1}, x_{u u}^{i-1}\right)$. In particular, the length of the curve $\Gamma^{i}=\operatorname{Image}\left(x^{i}\right)$ decreases along the discrete evolution generated by (4.1).

We end this section by discussing the full space-time discretization scheme to be used in all numerical simulations below. To derive the fully discrete analogue of (4.1) we use the uniform spatial grid $u_{j}=j h$ $(j=0, \ldots, m)$ with $h=1 / m$. The smooth solution $x$ is then approximated by the discrete values $x_{j}^{i}$ corresponding to $x(j h, i \tau)$. Using quite natural finite difference approximations of spatial differential terms in (4.1) we end up with the following semi-implicit difference scheme

$$
\frac{1}{2}\left(g_{j}^{i-1}+g_{j+1}^{i-1}\right) \frac{x_{j}^{i}-x_{j}^{i-1}}{\tau}=\frac{x_{j+1}^{i}-x_{j}^{i}}{g_{j+1}^{i-1}}-\frac{x_{j}^{i}-x_{j-1}^{i}}{g_{j}^{i-1}}, \quad i=1, \ldots, n, j=1, \ldots, m,
$$

where

$$
g_{j}^{i-1}=h_{j}^{i-1} \sqrt{\frac{k_{j}^{i-1}+\varepsilon}{\beta\left(k_{j}^{i-1}+\varepsilon\right)}}
$$

and

$$
\begin{aligned}
h_{j}^{i-1} & =\left|x_{j}^{i-1}-x_{j-1}^{i-1}\right|, \\
k_{j}^{i-1} & =\frac{\left|\arccos \left(\left(x_{j+1}^{i-1}-x_{j-1}^{i-1}, x_{j}^{i-1}-x_{j-2}^{i-1}\right) /\left(\left|x_{j+1}^{i-1}-x_{j-1}^{i-1}\right|\left|x_{j}^{i-1}-x_{j-2}^{i-1}\right|\right)\right)\right|}{h_{j}^{i-1}} .
\end{aligned}
$$

The scheme is subject to the periodic boundary conditions $x_{j+m}^{i}=x_{j}^{i}(j=-1,0,1)$. In each discrete computational time step $i \tau$ the scheme (4.6) leads to solving of two tridiagonal systems for the new curve position, which are computed in a very fast way. Let us mention that (4.6) does not involve the spatial grid parameter $h$ and in the linear case $\beta(k)=k$ it coincides with Dziuk's scheme [14]. 


\section{Discussion on numerical experiments}

Now, we present numerical results obtained by the approximation scheme (4.1) in the fully discrete version (4.6).

It follows from (2.4) that a special solution of $(0.1)$ with $\beta(k)=k^{m}, m>0$, is a circle homothetically shrinking to the center; its radius $R(t)$ being given by $R(t)=\left(R(0)^{m+1}-(m+1) t\right)^{1 /(m+1)}$. Using this formula we obtain exact blow up time for curvature. Table 1 shows relationship between exact and numerically computed blow up times for the power like function $\beta(k)=k^{m}$ for various $m>0$. It shows the exact blow up times $T_{\max }$ (see (2.5)), and numerically computed ones for time steps $\tau=0.01$, $\tau=0.001, \tau=0.0001$, respectively. The equidistant time step is used until the curvature begins to growth beyond a threshold value. After this moment we adaptively refine the time step to obtain numerical blow up (curvature of order $10^{5}$ ). We use the mesh containing 100 space grid points in order to represent the position of the curve.

In the case $\beta(k)=k^{1 / 3}$, arbitrary ellipse is a homothetic solution (see [2,28] and Section 2 of this paper). This property is also confirmed by our numerical simulations. During the time evolution the ratio $a / b$ of halfaxes stays constant up to the moment very close to the exact time of shrinking. The shape selfsimilarity during the evolution is justified by computing the isoperimetric ratio Iso $=L^{2} /(4 \pi S)$,

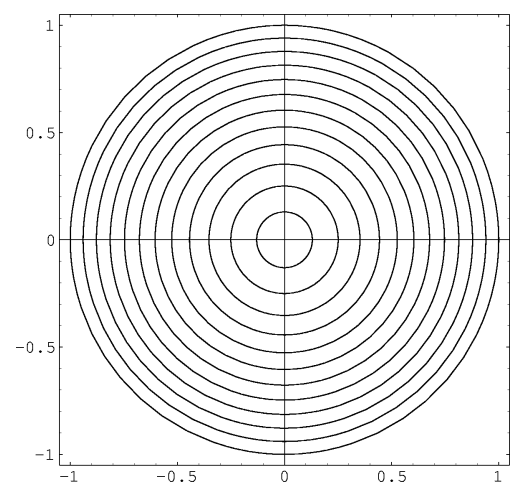

Fig. 1. Shrinking of the unit circle by nonlinear curve shortening with $\beta(k)=k^{1 / 2}$; numerical blow up time for the curvature is 0.671 , plotting time step is 0.1 .

Table 1

Relationship between exact and numerically computed curvature blow up times for initial unit circle, $\beta(k)=k^{m}$

\begin{tabular}{ccccccl}
\hline$m$ & $\frac{1}{10}$ & \multicolumn{1}{c}{$\frac{1}{4}$} & \multicolumn{1}{c}{$\frac{1}{3}$} & \multicolumn{1}{c}{$\frac{1}{2}$} & \multicolumn{1}{c}{1} & \multicolumn{1}{c}{0} \\
\hline$T_{\max }$-exact & $0.9 \overline{09}$ & 0.8 & 0.75 & $0.6 \overline{6}$ & 0.5 & 1.0 \\
$\tau=0.01$ & 0.942 & 0.835 & 0.785 & 0.701 & 0.536 & 1.02 \\
$\tau=0.001$ & 0.913 & 0.8047 & 0.754 & 0.671 & 0.5048 & 1.003 \\
$\tau=0.0001$ & 0.9098 & 0.8007 & 0.7506 & 0.6673 & 0.5005 & 1.0007 \\
\hline
\end{tabular}




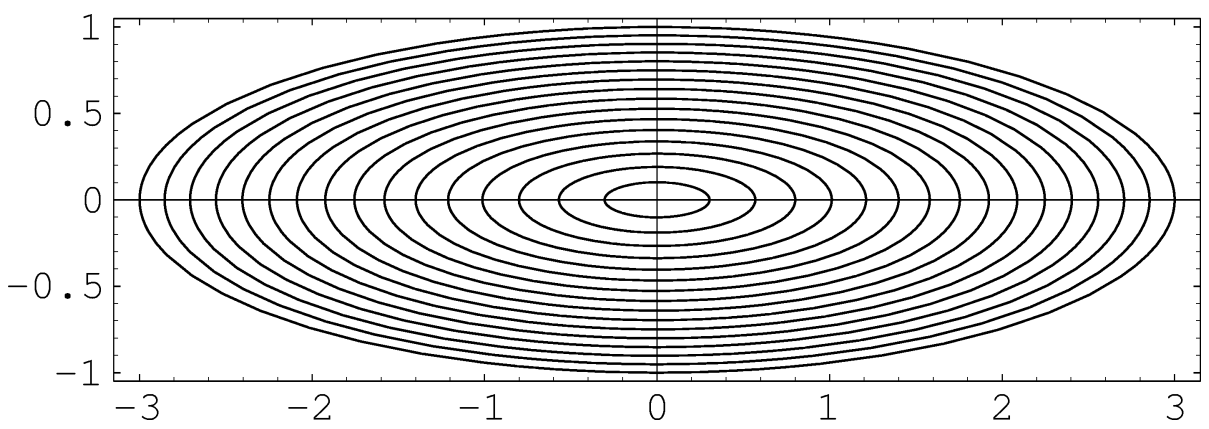

Fig. 2. Affine invariant evolution of the ellipse, initial halfaxes $a=3, b=1$; halfaxes ratio and isoperimetric ratio are conserved during the computations.

Table 2

Relationship between exact and numerically computed curvature blow up times for initial ellipses; $\beta(k)=k^{1 / 3}$

\begin{tabular}{cccc}
\hline Halfaxes ratio & $2: 1$ & $3: 1$ & $4: 1$ \\
\hline$T_{\max }$-exact & 1.191 & 1.560 & 1.890 \\
$\tau=0.001$ & 1.195 & 1.564 & 1.893 \\
Iso & 1.188 & 1.508 & 1.864 \\
\hline
\end{tabular}

Table 3

Evolution of the isoperimetric ratio

\begin{tabular}{ccccccc}
\hline Time $/$ Iso & 0 & 0.6 & 1 & 1.4 & 1.5 & 1.519 \\
\hline$\beta(k)=k^{1 / 2}$ & 1.508 & 1.36 & 1.25 & 1.11 & 1.07 & 1.04 \\
$\beta(k)=k^{1 / 4}$ & 1.508 & 1.63 & 1.79 & 2.39 & 3.36 & 4.12 \\
\hline
\end{tabular}

where $L$ is the length of the curve and $S$ is the enclosed area. It turns out that also this quantity is practically constant for the numerical solution.

For $m=\frac{1}{3}$, the exact blow up time for a shrinking ellipse can be computed (see Example 2.1) and is equal to $\frac{3}{4}(a b)^{2 / 3}$. In the next table we compare the numerical and exact blow up times for several ellipses. The ratio of halfaxes is printed in the headline of Table 2. The mesh containing 200 space grid points has been used for discretization of the curve. We also print the isoperimetric ratio which is conserved up to 4 digits during numerical evolution.

In spite of conservation of the isoperimetric ratio for $m=\frac{1}{3}$, it tends to 1 in numerical computations with $m=\frac{1}{2}$ and to $\infty$ for $m=\frac{1}{4}$, respectively. We print values of Iso $_{t}, t \in\left[0, T_{\max }\right)$, in these two cases for initial ellipse with halfaxes ratio $3: 1$.

In Figs. 3 and 4 we present the comparison of the numerical results obtained by two rather different methods. Namely, the tested method (4.1), based on the computing of the curve's position vector, and the method introduced in [23], based on the computing of the curvature of evolving curve. In the second case, the real motion is reconstructed from the computed curvature in discrete time steps. This method is based 


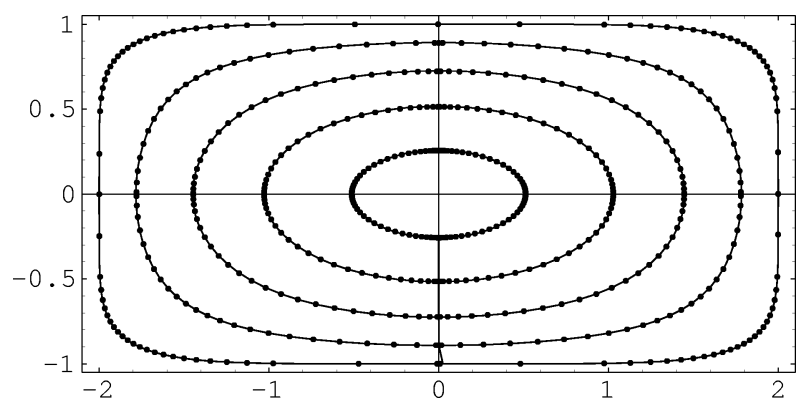

Fig. 3. Comparison of two different methods for evolution of convex curve: tick marks—method (4.1); solid lines-method from [23].

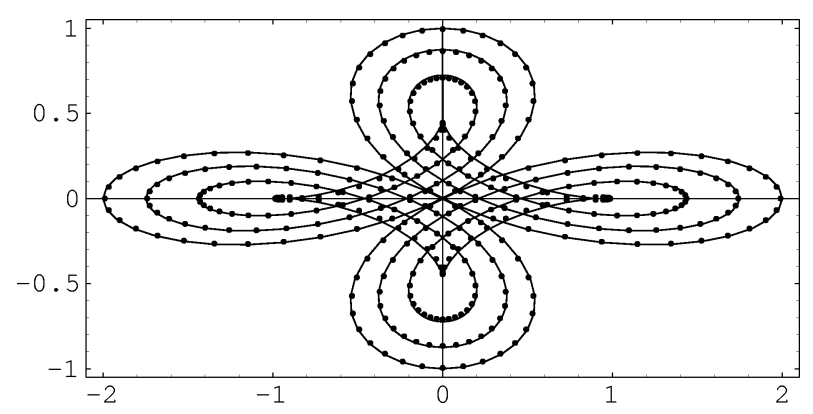

Fig. 4. Comparison of two different methods for evolution of selfintersecting "convex" curve: tick marks-method (4.1); solid lines-method from [23]. The evolving curve is plotted at the same discrete time moments until the "hair" singularity is formed. The method from [23] cannot continue beyond singularity.

on solving the nonlinear parabolic equation of porous-medium type and its convergence for (0.1) is the consequence of the results of [24]. However, it is restricted to convex cases (including selfintersections).

In Fig. 3 one sees suitable redistribution (from initial to the first plotted step) of computational grid points due to the presence of the tangential component of the velocity (see (1.4)). Due to the shape of $\beta$ the points with high curvatures are moving along the curves $(m<1)$ and it works against the degeneracy of equation. In spite of this, the effect is opposite for $m>1$ and leads to serious computational difficulties in that case. This phenomenon can be explained, in a satisfactory manner, by Eq. (1.4). It follows from (1.4) and (1.5) that the tangential velocity is proportional to $k_{s}$ times the sign of $m-1$. Therefore in the case $m \leqslant 1$ the tangential component of the velocity drives the grids away from the pieces of the curve with increasing curvature whereas its action is opposite in the case $m>1$.

The results discussed above are very accurate already for reasonable large computational time steps. It indicates the usefulness and effectiveness of the method even in cases when no exact solutions are known. In Figs. 5-8 we show evolutions of several initially nonconvex curves with the different choices of $\beta$. We also present the passage through singularities in some examples of immersed curves. A simple point removing algorithm has been built into the scheme preventing the " $\left|x_{u}\right|=0$ " kind of singularity and, moreover, it is very useful tool in order to pass through singularities and other situations when the grid points representing the curve move very close to each other. 


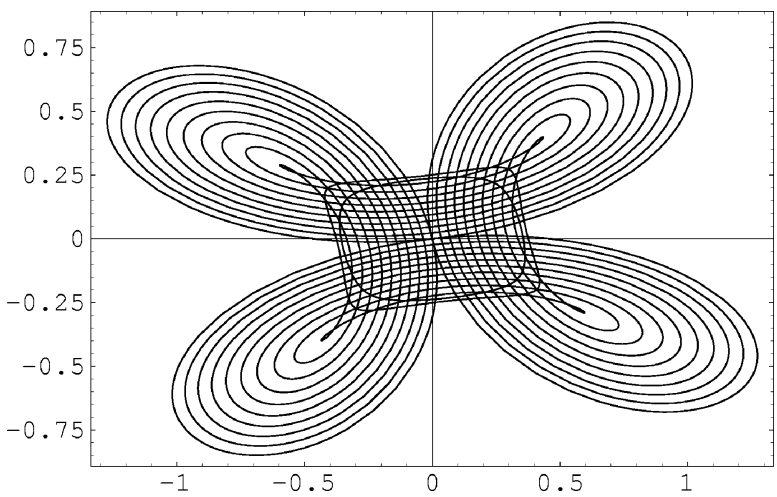

Fig. 5. Evolution of the affine transformed 4-petal through singularities to an ellipse-rounded point, $\beta(k)=k^{1 / 3}$.

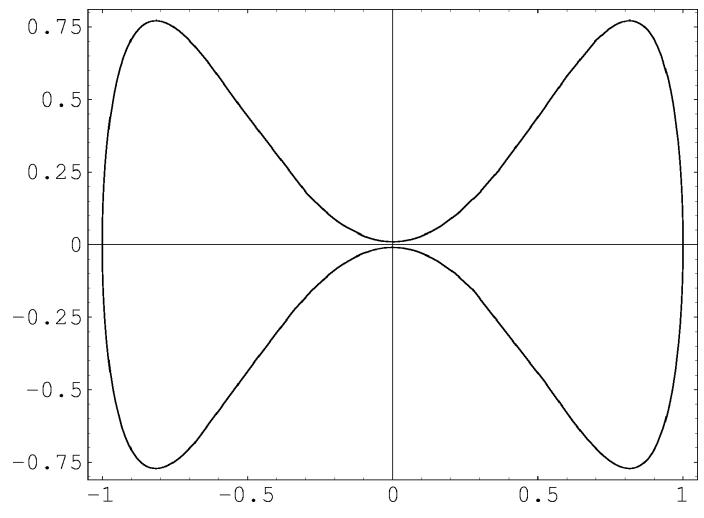

Fig. 6. The initial nonconvex curve.

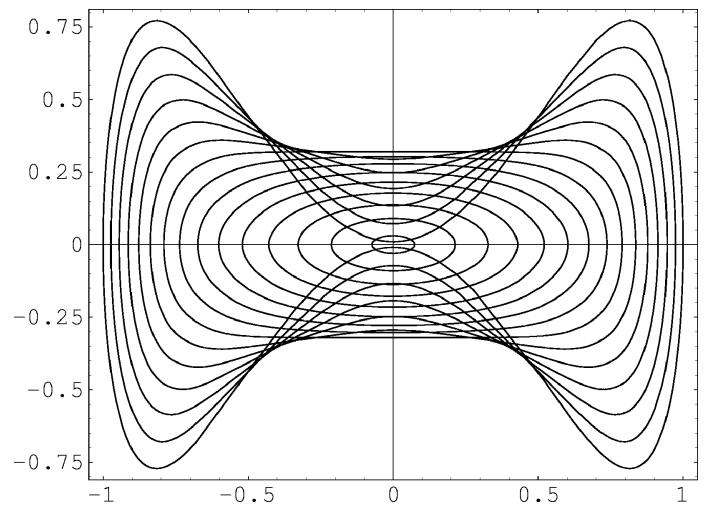

Fig. 6b. The case $\beta(k)=k^{1 / 3}$. Time interval is $[0,0.56]$.

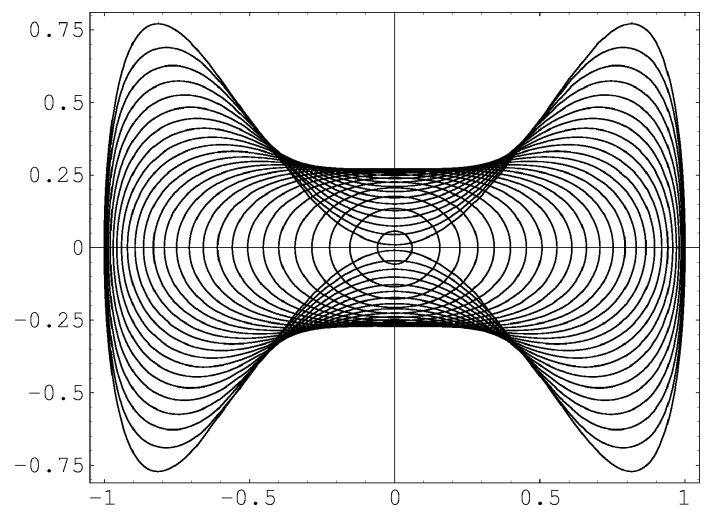

Fig. 6a. The case $\beta(k)=k$. Time interval is $[0,0.26]$.

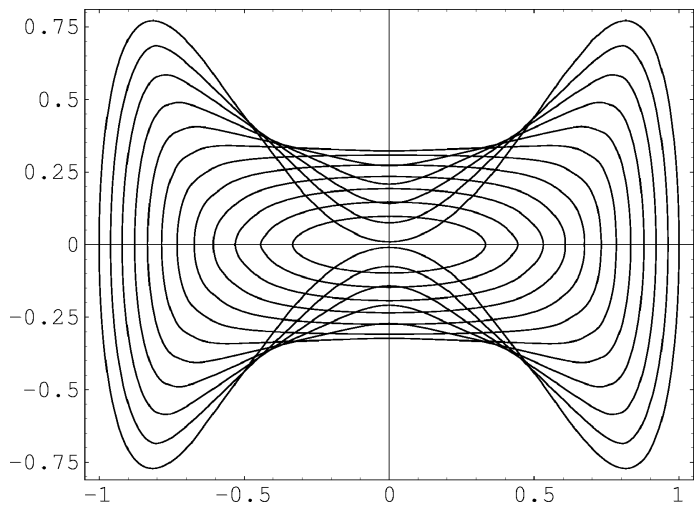

Fig. 6c. The case $\beta(k)=k^{1 / 5}$. Time interval is $[0,0.56]$. 


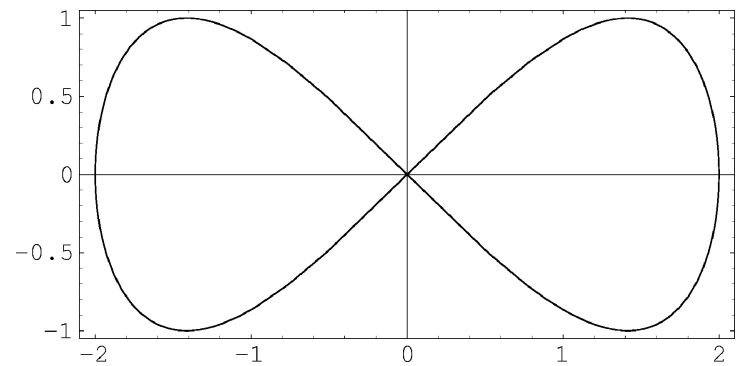

Fig. 7. The initial $\infty$-like selfintersecting curve.

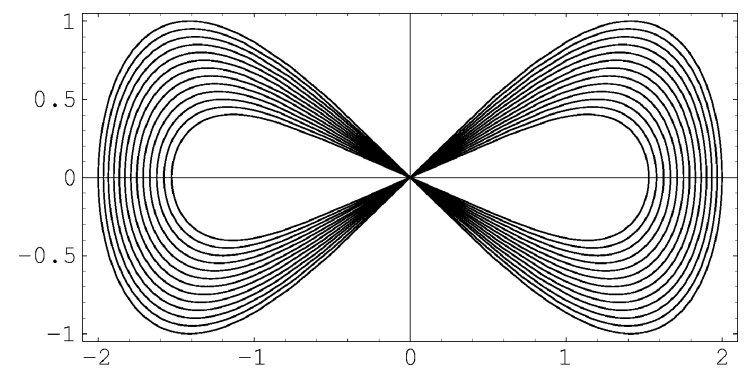

Fig. 7b. The case $\beta(k)=k^{1 / 3}$. Time interval is $[0,0.3]$.

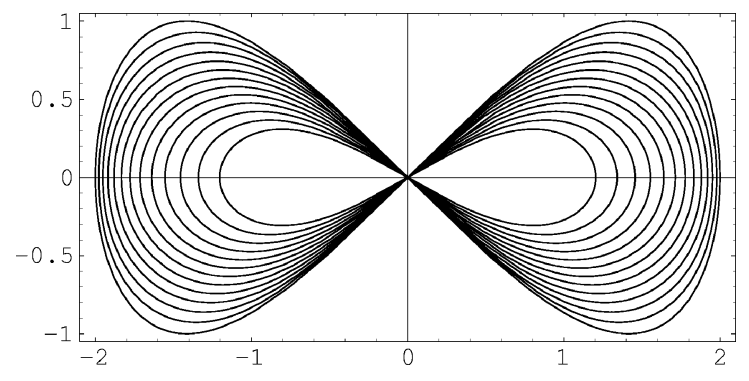

Fig. 7a. The case $\beta(k)=k$. Time interval is $[0,0.3]$.

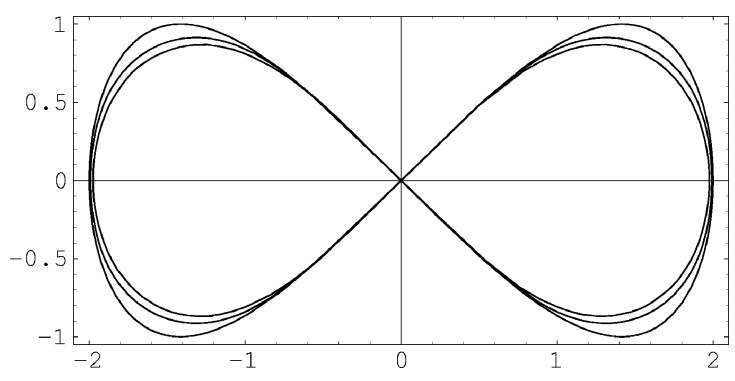

Fig. 7c. The case $\beta(k)=k^{2}$. Time interval is $[0,0.08]$.

Figs. 6a-6c show the time evolution of the initial curve depicted in Fig. 6. The plotting step is 50 . The initial curve has the parameterization $\left\{\left(\cos (2 \pi u), 2 \sin (2 \pi u)-1.99 \sin (2 \pi u)^{3}\right), u \in[0,1]\right\}$ and the spatial mesh contained 100 equally distributed points. In Fig. $6 \mathrm{a}$, respectively $6 \mathrm{~b}$, with $\beta(k)=k$, respectively $\beta(k)=k^{1 / 3}$, one can see the evolution of a nonconvex curve to a circle-rounded point, respectively to an ellipse-rounded point, in Fig. $6 \mathrm{c}$ we have plotted evolution of the nonconvex curve with the blow up of isoperimetric ratio for the case $\beta(k)=k^{1 / 5}$.

Figs. $7 \mathrm{a}-7 \mathrm{c}$ show the time evolution of the initial curve depicted in Fig. 7. The plotting step is 40 (Figs. 7a, 7b) and 30 (Fig. 7c). The initial curve has the parametrization $\{(2 \cos (2 \pi u), \sin (4 \pi u)), u \in$ $[0,1]\}$ and the spatial mesh contained 100 equally distributed points. In Fig. 7c with $\beta(k)=k^{2}$ one sees very different behavior for the parts of curve with curvature close or equal to 0 in comparison with Fig. 7b, where $\beta(k)=k^{1 / 3}$. We did not trace the evolution of curves for $\beta(k)=k^{2}$ beyond the time interval $[0,0.1]$ as it becomes unstable for large time intervals of simulation. The pieces of the curve with the curvature close to zero do not move for a long time in the case $\beta(k)=k^{2}$ (see Fig. 7c) whereas they move quickly apart from each other in the case $\beta(k)=k^{1 / 3}$ (see Fig. 7b). This phenomenon can be related to the effect of the the slow and fast diffusion diffusion effect known from the analysis of porous medium equations.

Another example of the time evolution of an irregular initial curve with the large variation in the curvature is demonstrated by Figs. 8a-8c with initial curve depicted in Fig. 8. We have used the time step $\tau=0.001$, plotting step 50, and the mesh contained 200 grids. 


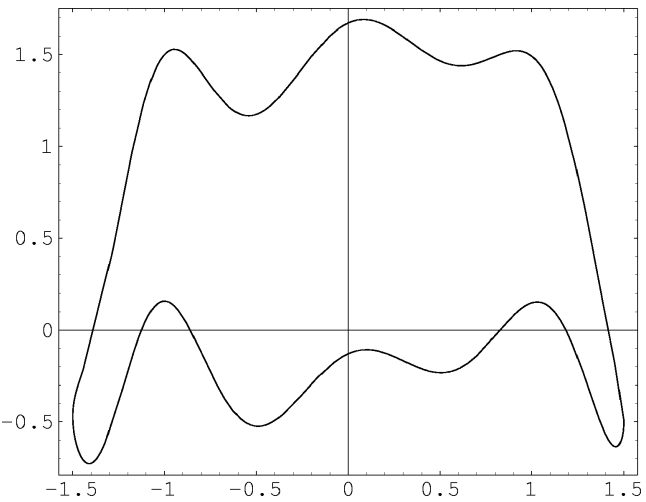

Fig. 8. The initial irregular plane curve.

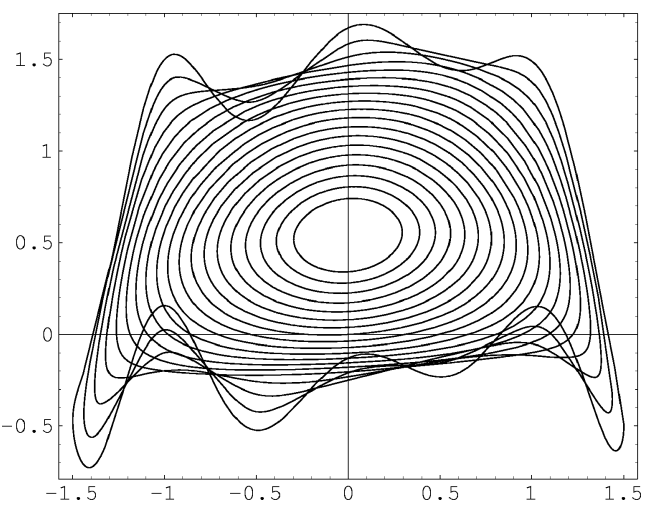

Fig. $8 \mathrm{~b}$. The case $\beta(k)=k^{1 / 3}$. Time interval is $[0,0.9]$.

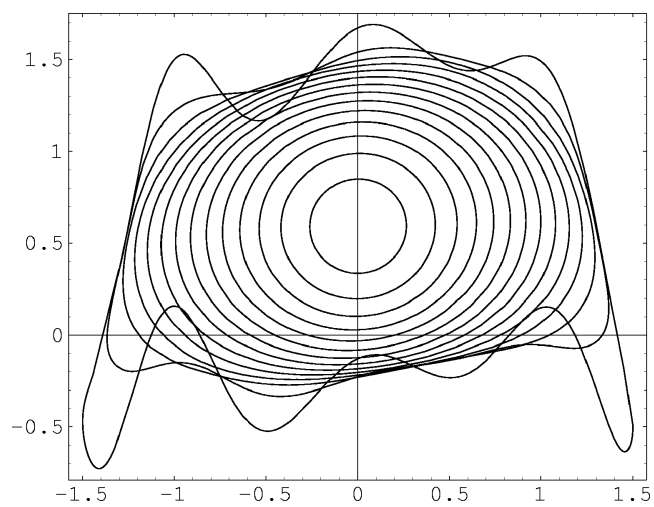

Fig. 8a. The case $\beta(k)=k$. Time interval is $[0,0.65]$.

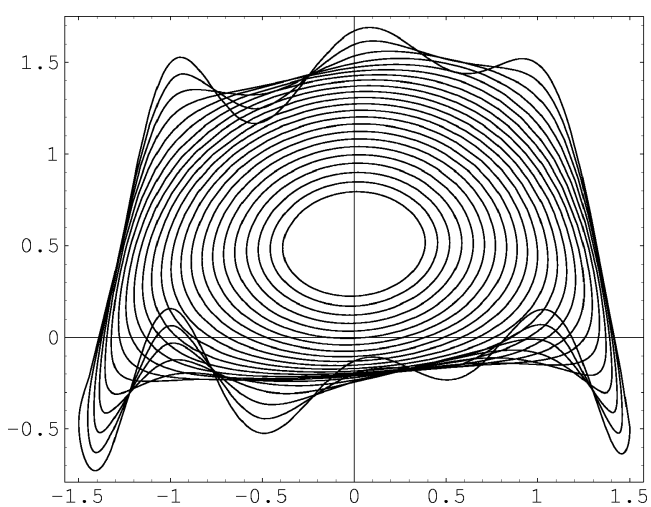

Fig. 8c. The case $\beta(k)=\arctan (k)$. Time interval is $[0,1.1]$.

\section{References}

[1] U. Abresch and J. Langer, The normalized curve shortening flow and homothetic solutions, J. Differential Geometry 23 (1986) 175-196.

[2] L. Alvarez, F. Guichard, P.L. Lions and J.M. Morel, Axioms and fundamental equations of image processing, Arch. Rat. Mech. Anal. 123 (1993) 200-257.

[3] L. Alvarez and J.M. Morel, Formalization and computational aspects of image analysis, Acta Numer. (1994) $1-59$.

[4] S.B. Angenent, Nonlinear analytic semiflows, Proc. Roy. Soc. Edinburgh Sect. A 115 (1990) 91-107.

[5] S.B. Angenent, Parabolic equations for curves on surfaces I: Curves with $p$-integrable curvature, Ann. Math. 132 (3) (1990) 451-483.

[6] S.B. Angenent, Parabolic equations for curves on surfaces II: Intersections, blow-up and generalized solutions, Ann. Math. 133 (1) (1991) 171-215.

[7] S.B. Angenent, On the formation of singularities in the curve shortening flow, J. Differential Geometry 33 (1991) 601-633. 
[8] S.B. Angenent and M.E. Gurtin, Multiphase thermomechanics with an interfacial structure 2. Evolution of an isothermal interface, Arch. Rat. Mech. Anal. 108 (1989) 323-391.

[9] S.B. Angenent and M.E. Gurtin, Anisotropic motion of a phase interface. Well-posedness of the initial value problem and qualitative properties of the interface, J. Reine Angew. Math. 446 (1994) 1-47.

[10] S. Altschuler, Singularities of the curve shrinking flow for space curves, J. Differential Geometry 34 (1991) 491-514.

[11] S. Altschuler and M.A. Grayson, Shortening space curves and flow through singularities, J. Differential Geometry 35 (1992) 283-298.

[12] Y.-G.Chen, Y. Giga and S. Goto, Uniqueness and existence of viscosity solutions of generalized mean curvature flow equation, J. Differential Geometry 33 (1991) 749-786.

[13] M.G. Crandall, H. Ishii and P.L. Lions, User's guide to viscosity solutions of second order partial differential equations, Bull. Amer. Math. Soc. 27 (1992) 1-67.

[14] G. Dziuk, Convergence of a semi discrete scheme for the curve shortening flow, Math. Models Methods Appl. Sci. 4 (4) (1994) 589-606.

[15] G. Dziuk, Convergence of a scheme for anisotropic curvature flow, Abstract (from the Conference on Generalized Stefan Problem: Analysis and Numerical Methods, Pavia, 1995) in FBP News 9 (1995) 5.

[16] L.C. Evans and J. Spruck, Motion of level sets by mean curvature. I, J. Differential Geometry 33 (1991) 635-681.

[17] M. Gage and R.S. Hamilton, The heat equation shrinking convex plane curves, J. Differential Geometry 23 (1986) 285-314.

[18] M. Grayson, The heat equation shrinks embedded plane curves to round points, J. Differential Geometry 26 (1987) 285-314.

[19] J. Kačur and K. Mikula, Solution of nonlinear diffusion appearing in image smoothing and edge detection, Appl. Numer. Math. 17 (1995) 47-59.

[20] M. Kass, A. Witkin and D. Terzopoulos, Snakes: active contours models, in: Proc. 1st International Computer Vision Conference (1987) 259-269.

[21] P.L. Lions, Axiomatic derivation of image processing models, Math. Models Methods Appl. Sci. 4 (1994) 467-475.

[22] A. Mackworth and F. Mokhtarian, A theory of multiscale, curvature-based shape representation for planar curves, IEEE Trans. Pattern Anal. Machine Intell. 14 (1992) 789-805.

[23] K. Mikula, Solution of nonlinear curvature driven evolution of plane convex curves, Appl. Numer. Math. 21 (1997) 1-14.

[24] K. Mikula and J. Kačur, Evolution of convex plane curves describing anisotropic motions of phase interfaces, SIAM J. Sci. Comput. 17 (6) 1996.

[25] S. Osher and J. Sethian, Fronts propagating with curvature dependent speed: algorithm based on HamiltonJacobi formulation, J. Comput. Phys. 79 (1988) 12-49.

[26] S. Patankar, Numerical Heat Transfer and Fluid Flow (Hemisphere Publ. Corp., New York, 1980).

[27] B.M. ter Haar Romeny (Ed.), Geometry Driven Diffusion in Computer Vision (Kluwer, Dordrecht, 1994).

[28] G. Sapiro and A. Tannenbaum, On affine plane curve evolution, J. Funct. Anal. 119 (1) (1994) 79-120.

[29] A. Schmidt, Computation of three dimensional dendrites with finite elements, J. Comput. Phys. 125 (2) (1996) 293-312.

[30] J. Sethian, Numerical algorithm for propagating interfaces: Hamilton-Jacobi equations and conservation laws, J. Differential Geometry 31 (1990) 131-161. 